\title{
DESCRIPCIÓN DE UNA ESPECIE NUEVA DEL GÉNERO TETRALEURODES COCKERELL (HEMIPTERA: ALEYRODIDAE) DE MÉXICO
}

\author{
DESCRIPTION OF A NEW SPECIES OF GENUS TETRALEURODES \\ COCKERELL (HEMIPTERA: ALEYRODIDAE) FROM MEXICO
}

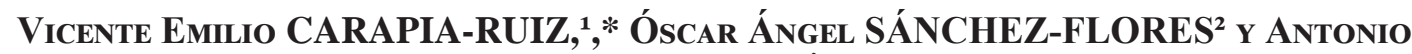 \\ CASTILLO-GUTIÉRREZ ${ }^{1}$
}

\begin{abstract}
${ }^{1}$ Universidad Autónoma del Estado de Morelos (UAEM), Escuela de Estudios Profesionales de Xalostoc (EESuX). Av. Nicolas Bravo s/n, Parque Industrial Cuautla, Xalostoc, Ayala, Morelos.

${ }^{2}$ Universidad Autonóma Agraria “Antonio Narro” < oscaruaaan@gmail.com>; <antoniocg62@hotmail.com>.

* Autor de correspondencia:<vcarapia@hotmail.com>.
\end{abstract}

Recibido: 10/08/2016; aceptado: 08/03/2017

Editor responsable: Jesús Romero Nápoles.

\begin{abstract}
Carapia-Ruiz, V. E., Sánchez-Flores, Ó. A. y Castillo-Gutiérrez, A. (2017). Descripción de una especie nueva del género Tetraleurodes Cockerell (Hemiptera: Aleyrodidae) de México. Acta Zoológica Mexicana (n.s.), 33(2), 243-250.
\end{abstract}

RESUMEN. El presente estudio reporta la descripción de Tetraleurodes tepalcingo sp. nov., encontrada en Morelos, México en tallos de Acacia farneciana (L) Willd. Se discute la relación de esta especie con Tetraleurodes caulicola Nakahara y Tetraleurodes tuberculosa Nakahara. Se proporciona una clave para la identificación de especies del género Tetraleurodes de México.

Palabra clave: Tetraleurodes, sp. nov., Aleyrodidae, moscas blancas, México.

\section{INTRODUCCIÓN}

Quaintance y Baker (1914) describieron el género Tetraleurodes incluyendo18 especies en el mundo, mencionando sólo a T. acaciae para México. Baker (1937) reportó para México a T. acaciae, T. fici, T. mori, T. pringlei, T. usorum. Posteriormente Sampson y Drews (1941) en un trabajo sobre moscas blancas de México describen a $T$. bidentatus, $T$. contigua, $T$. quadratus y T. truncatus; en tanto que Nakahara (1995) describió a T. caulicola, T. chivela, T. mexicana y T. quercicola, haciendo referencia de su presencia en México, este mismo autor describe otras especies más del género entre las que se encuentran T. bireflexa y T. melanops sin indicar su presencia en México. Martin (2005) estudiando los Aleyrodinae de Belice reportó la identificación de siete especies y mencionó a 17 no identificadas, de las cuales la mayoría podrían ser nuevas para la ciencia. En colectas realizadas a partir
Carapia-Ruiz, V. E., Sánchez-Flores, Ó. A., \& Castillo-Gutiérrez, A. (2017). Description of a new species of genus Tetraleurodes Cockerell (Hemiptera: Aleyrodidae) from Mexico. Acta Zoológica Mexicana (n.s.), 33(2), 243-250.

ABSTRACT. The present study reports the description of Tetraleurodes tepalcingo sp. nov., found in Morelos México on stems of Acacia farneciana (L.) Willd. The relationship of this species with Tetraleurodes caulicola Nakahara and Tetraleurodes tuberculosa Nakahara is discused. A puparial key to identify species of the genus Tetraleurodes from Mexico is provided.

Key words: Tetraleurodes, sp. nov., Aleyrodidae, Whiteflies, Mexico.

del 2002 en México se indicó la presencia de especies no conocidas como es el caso de una especie de género Tetraleurodes del grupo acaciae encontrada en Tepalcingo, Morelos México (Carapia et al., 2016). El presente estudio tiene como objetivo reportar la descripción de $T$. tepalcingo sp. nov., y proporcionar una clave para la identificación de especies del género Tetraleurodes encontradas en México.

\section{MATERIALES Y MÉTODOS}

Se realizaron montajes de especímenes (puparios) en portaobjetos para microscopio compuesto. La metodología seguida para la preparación de especímenes en portaobjetos fue la de Martin (2004) con algunas modificaciones.

1. Maceración de pupas en hidróxido de potasio al 40 $\%$ durante un lapso de 20 a 30 minutos en un vi- 
drio de reloj; para las cubiertas pupales, de las que emergieron los adultos, se siguió directamente el paso tres.

2. Decolorado en peróxido de hidrógeno hasta una tonalidad amarillenta y lavado de pupasen agua destilada.

3. Eliminación de cera de pupas en cloral-fenol (hidrato de cloral 1 parte: fenol 1 parte) por 30 minutos a $60^{\circ} \mathrm{C}$.

4. Deshidratado en ácido acético glacial por 5 min.

5. Tratamiento en aceite de clavo por dos horas o más.

6. Montaje en bálsamo de Canadá.

\section{Observaciones y mediciones de estructuras}

Las preparaciones se examinaron en un microscopio Motica BA 310 a 40, 100, 400 y 1000 X en el laboratorio de Entomología de la EESuX-UAEM. Los especímenes se estudiaron con ayuda de descripciones y claves de Sampson y Drews (1941) y Nakarara (1995). Las microfotografías de Martin (2005) fueron de gran utilidad para la determinación de la especie.

\section{RESULTADOS Y DISCUSIÓN}

Tetraleurodes tepalcingo Carapia-Ruiz sp. nov. (Figs. 1-15)

Cubierta pupal: Alargada-oval, margen anterior redondeado, margen posterior redondeado; submargen medianamente elevado; disco dorsal con relieves en cefalotórax y abdomen, más alto que el submargen. Dimensiones del margen: longitud $1.35-1.38 \mathrm{~mm}$, ancho $0.98-99 \mathrm{~mm}$, de color negro; cera ausente en el disco dorsal pero con una franja de cera blanca en el margen.

Margen: Dientes marginales cortos de 10-12 $\mu \mathrm{m}$ de largo ocupando 6 en $100 \mu \mathrm{m}$ aparentemente lobulados, con tubérculos desde la base hasta su ápice lo que le da una forma irregular (Fig. 3), seta marginal anterior 20-25 $\mu \mathrm{m}$; marginal posterior 50-60 $\mu \mathrm{m}$ de largo.

Submargen: Situado entre el disco dorsal y el ventral diferenciando dos pliegues uno ligero cerca de la línea de dientes marginales y la otra más profunda próxima a la línea del submargen ambos pliegues ausentes en la parte anterior y posterior (Figs. 2, 4, 7, 8). Con cerca de 36 pares de tubérculos glandulares de 9-12 $\mu \mathrm{m}$ de ancho en línea casi uniforme en alineamiento como en distancia- miento excepto en la región anterior y posterior donde no se presentan (Figs. 1, 4). Surcos transversos con tubérculos entre el margen y los tubérculos glandulares; surcos sin tubérculos entre los tubérculos glandulares y la línea submarginal; cerca de un surco por diente marginal cerca del margen y aproximadamente dos cerca de los tubérculos glandulares. Cada diente con un glandular pálido en forma de "u" cerca del área marginal (Fig. 5). Seta caudal de 45-50 $\mu \mathrm{m}$ de largo casi al nivel de la seta marginal posterior (Fig. 8).

Disco dorsal (Figs. 1, 2): Superficie con esculpido característico (Figs. 6-12), subdorso reflejado del submargen en su valor más alto. Sutura longitudinal de la muda de 520-530 $\mu \mathrm{m}$ extendiéndose hasta el margen anterior; sutura transversa de la muda de 510-520 $\mu$ m extendida hasta la línea del submargen, terminando al nivel de la sutura metatorácica; margen de las suturas de la muda lisas; sutura mesotorácica llega al subdorso, no alcanza la línea submarginal. Marcas oculares ausentes. Seta cefálica 17-20 $\mu \mathrm{m}$ de largo, seta mesotorácica de 18-20 $\mu \mathrm{m}$ de largo (Fig. 11), seta metatorácica de 17-20 $\mu \mathrm{m}$ de largo (Fig. 11), octava seta abdominal 15-18 $\mu \mathrm{m}$ de largo, anterolaterada del orificio vasiforme, más cerca del orificio vasiforme que de la sutura del VIII segmento abdominal. Tres áreas elevadas en la región cefalotorácica; segmentos abdominales I-VIII con un par de depresiones en la parte media abdominal de 20 por $50 \mu \mathrm{m}$ (Fig. 9). Poros del disco dorsal de $3 \mu \mathrm{m}$ de diámetro distribuidos, siempre en conjunto de dos el poro ligeramente mayor y el porete ligeramente menor, como sigue: 10 cefálicos medianos (Figs. 7, 10), 10 cefálicos subdorsales, dos protorácicos medianos, cuatro protorácicos subdorsales, dos mesotorácico medianos, cuatro mesotorácicos subdorsales, dos metatorácico medianos, cuatro metatorácicos subdorsales, cuatro a seis en los segmentos abdominales I-III, normalmente 10 en los segmentos IV-VI, en los segmentos abdominales VII-VIII cuatro a seis. Segmento abdominal I normalmente con un tubérculo medio de $25 \mu \mathrm{m}$ de diámetro (Fig. 6); líneas intersegmentales bien definidas entre la región media dorsal y la mitad del subdorso; área media de los segmentos abdominales 1-VI 60-70 $\mu \mathrm{m}$, del segmento VII $50 \mu \mathrm{m}$, del segmento VIII (hasta el margen anterior del orificio vasiforme) $70 \mu \mathrm{m}$, distancia del orificio vasiforme al margen $110 \mu \mathrm{m}$. Orificio vasiforme semicordiforme a semioval de 60-65 $\mu \mathrm{m}$ de largo y 64-70 $\mu \mathrm{m}$ de ancho, Opérculo semicordiforme cubriendo completamente al orificio. Lingula cubierta por el opérculo, mitad apical agrandada de forma oval con espínulas. Surco caudal ausente, puede confundir el inicio 

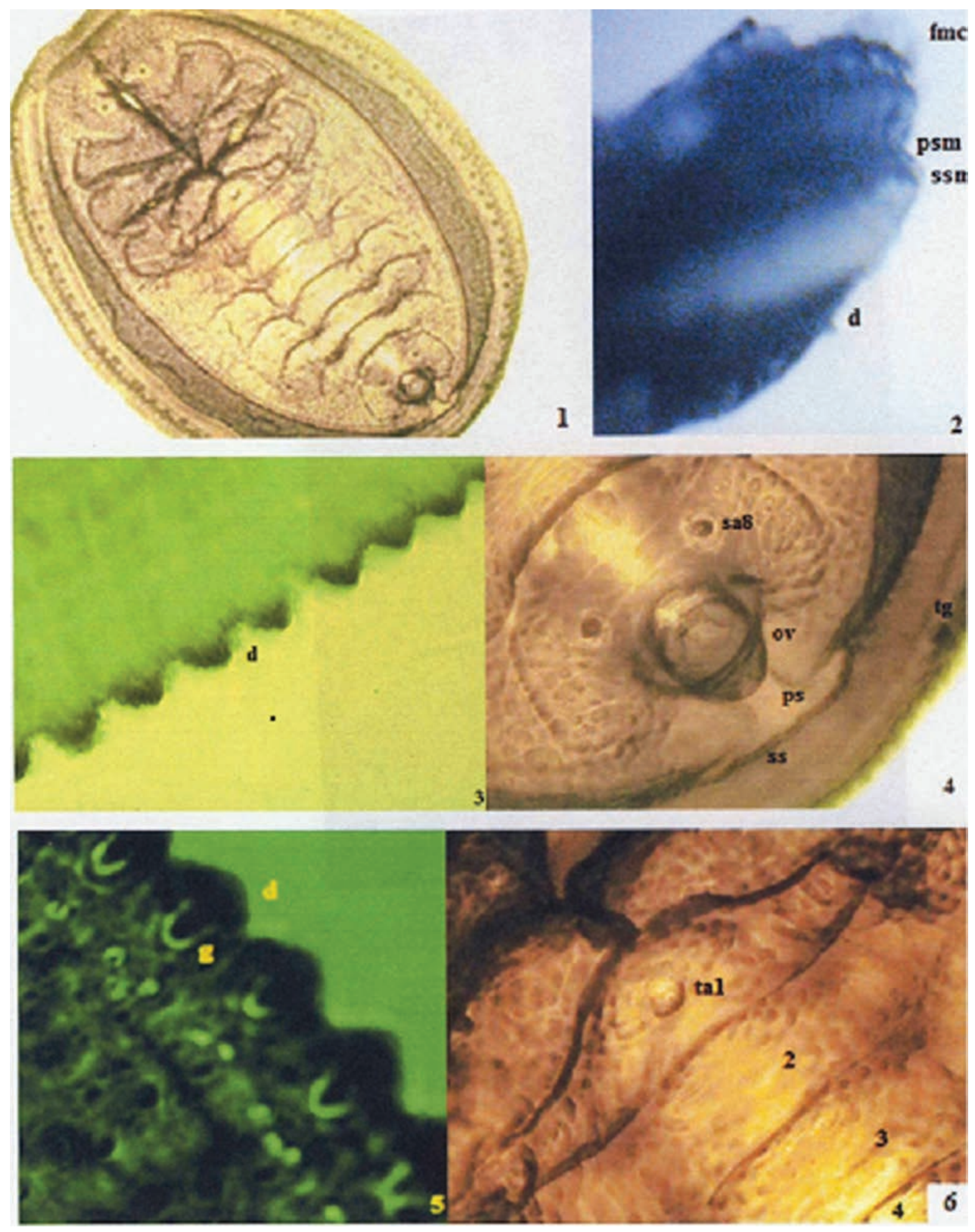

Figuras 1-6. Tetraleurodes tepalcingo Carapia-Ruiz sp. nov. 1) Cubierta pupal. 2) Cubierta pupal sin procesar mostrando el pliegue sobmarginal (psm), la franja marginal de cera (fmc), sutura submarginal (ssm). 3) Margen, dientes (d) mostrando sus tubérculos. 4) Parte posterior, tubérculo glandular (tg), orificio vasiforme (ov), sutura submarginal (ss), término del pliegue submarginal (ps), base setal del segmento abdominal VIII (sa8). 5) Dientes marginales (d) glándulas (g) en la base de los dientes marginales. 6) Área dorsal media, tubérculo medio en segmento abdominal I (ta1). 

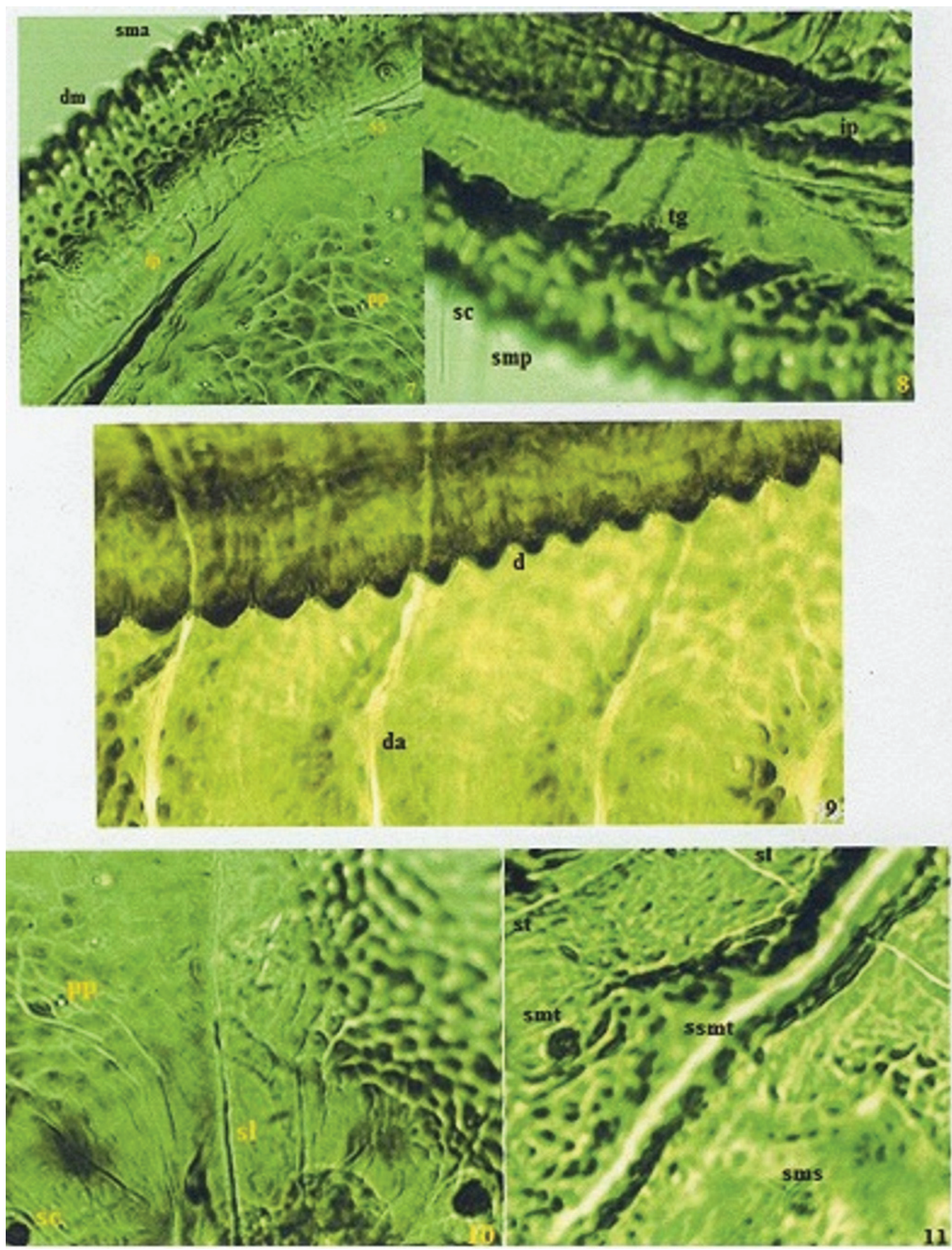

Figuras 7-11. Tetraleurodes tepalcingo Carapia-Ruiz sp. nov. 7) Área cefálica, seta marginal anterior (sma), sutura submarginal (ss), inicio del pliegue submarginal (ip), poro y porete (pp). 8) Área posterior, seta caudal (sc), seta marginal posterior (smp), término del pliegue submarginal (ip), tubérculo glandular (tg). 9) Depresiones abdominales medianas (da). 10) Región cefálica, seta cefálica (sc), sutura longitudinal de la muda (sl), poros y poretes (pp). 11) Meso-metatórax, sutura transversa de la muda (st), sutura longitudinal de la muda (sl), sutura metatorácica (ssmt), seta metatorácica (smt), seta mesotorácica (sms). 

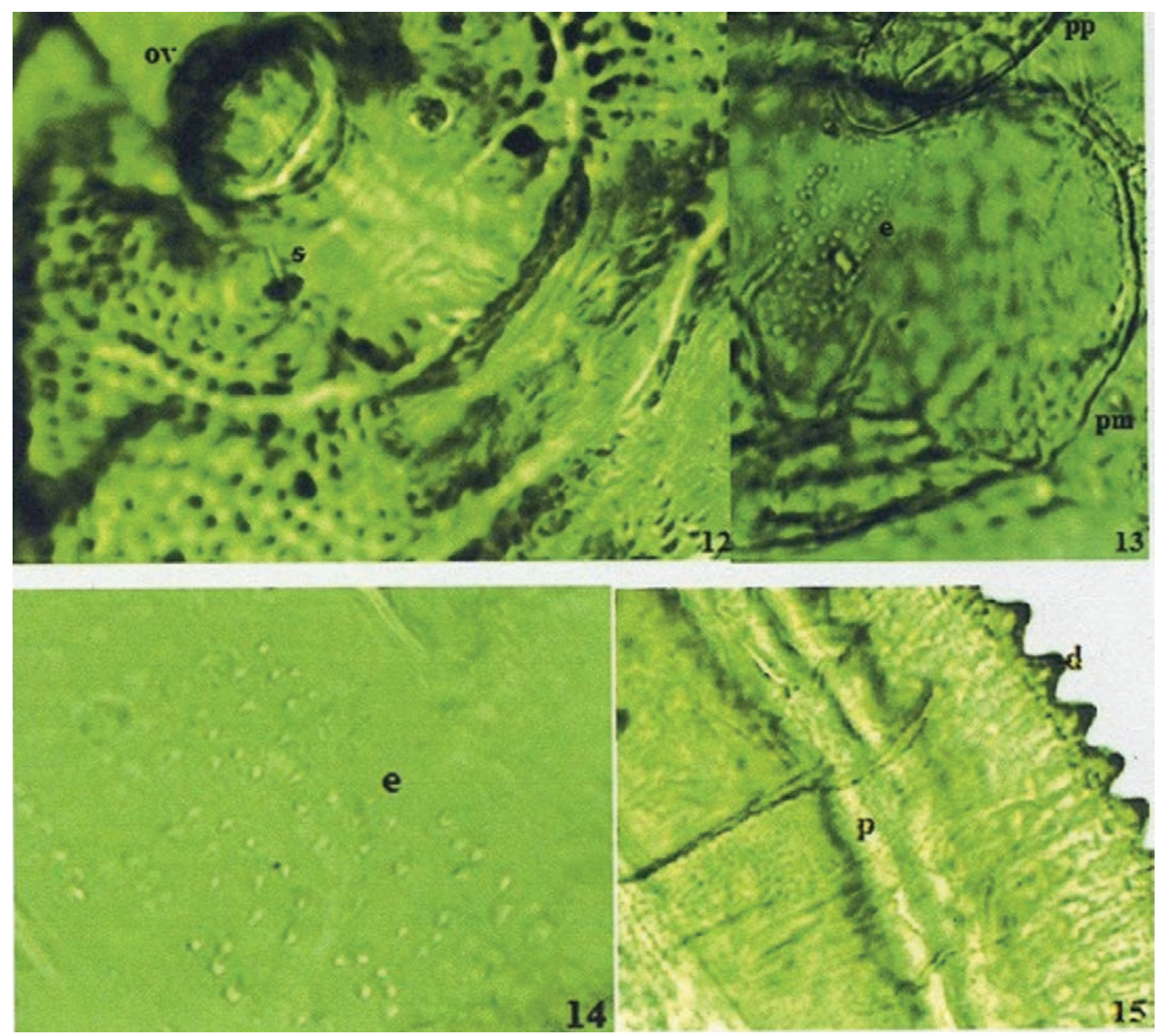

Figuras 12-15. Tetraleurodes tepalcingo Carapia-Ruiz sp. nov. 12) Segmento abdominal VIII, seta del segmento abdominal VIII (s). 13) Patas torácicas, espínulas (e), pata protorácica (pp), pata mesotorácica (pm). 14) Base de pata mesotorácica, espínula (e). 15) Pliegue toracico traqual (p), dientes (d).

del pliegue del submargen al nivel del orificio vasiforme (Fig. 4).

Vientre: Antena extendiéndose hasta el espiráculo anterior. Pliegues traqueales abdominales y torácicos bien definidos, 40-160 $\mu \mathrm{m}$ de ancho y 20-22 $\mu \mathrm{m}$ de largo, con granulaciones característica (Fig. 15). Patas mesotorácicas y metatorácicas con microseta. Espínulas pequeñas en la base de las patas (Fig. 13, 14). Sacos adhesivos bien desarrollados. Octava seta abdominal 30-35 $\mu \mathrm{m}$ de largo. Holotipo: En tallos de Acacia farnesiana (L.) Willd. 14marzo-1991. Tepalcingo, Morelos México, depositado en CNIN Instituto de Biología, UNAM, Ciudad de México. Paratipos, 2, mismos datos del holotipo y depositados en la misma colección, tres depositados en la colección del autor. Cinco en tallos de Acacia farnesiana (L.) Willd. 30-junio-1991. Tlayecac, Ayala, Morelos México, dos depositados en la colección del Museo Bohart de la Universidad de California, Davis, Ca, USA (UCD) y tres en la colección del autor.

Hospedero: Sobre tallos de Acacia farnesiana (L.) Willd. (Vachellia farnesiana (L.) Wight \& Arn.) (Figs. 1, 2)

Distribución: Municipios de Tepalcingo y Ayala del Estado de Morelos, México. Es posible que se encuentre en otros lugares, al menos en el centro de México.

Etimología: el nombre especifico Tetraleurodes tepalcingo Carapia-Ruiz sp. nov., es referido a la región del 
municipio de Tepalcingo, Morelos, lugar donde se encontró la mayor cantidad de especímenes por planta.

Discusión: Tetraleurodes tepalcingo Carapia-Ruiz sp. nov., es la especie de mayor tamaño de Tetraleurodes grupo acaciae caracterizado por tener tubérculos glandulares en el submargen. En esta especie la sutura del octavo segmento abdominal no se dirige lateralmente como en Tetraleurodes caulicola Nakahara sino su dirección es hacia la parte caudal; también infestan tallos de leguminosa. El tamaño considerablemente mayor y la carencia de marcas oculares cefalicas permite separar y definir $\mathrm{Te}$ traleurodes tepalcingo Carapia-Ruiz sp. nov., de T. tuberculosa Nakahara.

\section{Clave para especies del género Tetraleurodes de México}

1 Tubérculos glandulares presentes en el submargen; usualmente sobre leguminosas . . . . . . . . . . . 4 - Sin tubérculos glandulares en el submargen; sobre varios hospederos . .................... 2 Con una banda de cerca de cuatro papilas en el submargen (Fig. 18) ........ T. contigua (Sampson y Drews) - Sin una banda de cerca de cuatro papilas en el submar-

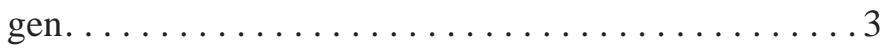
3 Dientes del submargen truncado (Fig. 17).............. T. truncatus Sampson y Drews - Dientes del submargen no truncados ...........8 8 4 Segmento abdominal VIII no separado de los segmentos anteriores, línea intersegmental VII-VIII no se prolonga hasta el pliegue submarginal; cubiertas pupales alargada. ............................. 5 - Segmento abdominal VIII separado de los segmentos anteriores, línea intersegmental VII-VIII se prolonga hasta el pliegue submarginal; cubiertas púpales oval. . . . . 6 5 Banda microtubercular submarginal no sobrepasa los tubérculos glandulares submarginales (Figs. 1, 4, 7,

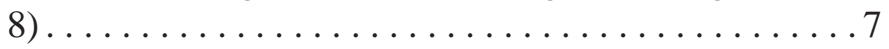
- Banda microtubercular submarginal sobrepasa los tubérculos glandulares submarginales (Figs. 23, 24).................. T. bireflexa Nakahara 6Cubiertapupalmás1.3mmdelargoy de98-1 mmdeancho, con tubérculos en la superficie dorsal más o menos uniforme, octava seta abdominal más cerca del orificio que de la sutura VIII, la cual se prolonga hacia la parte posterior(Figs. 1-15) .......... T. tepalcingo Carapia-Ruiz sp. nov. - Cubierta pupal menor a $1.3 \mathrm{~mm}$ de largo, con dientes en las depresiones abdominales y torácicas, oc-

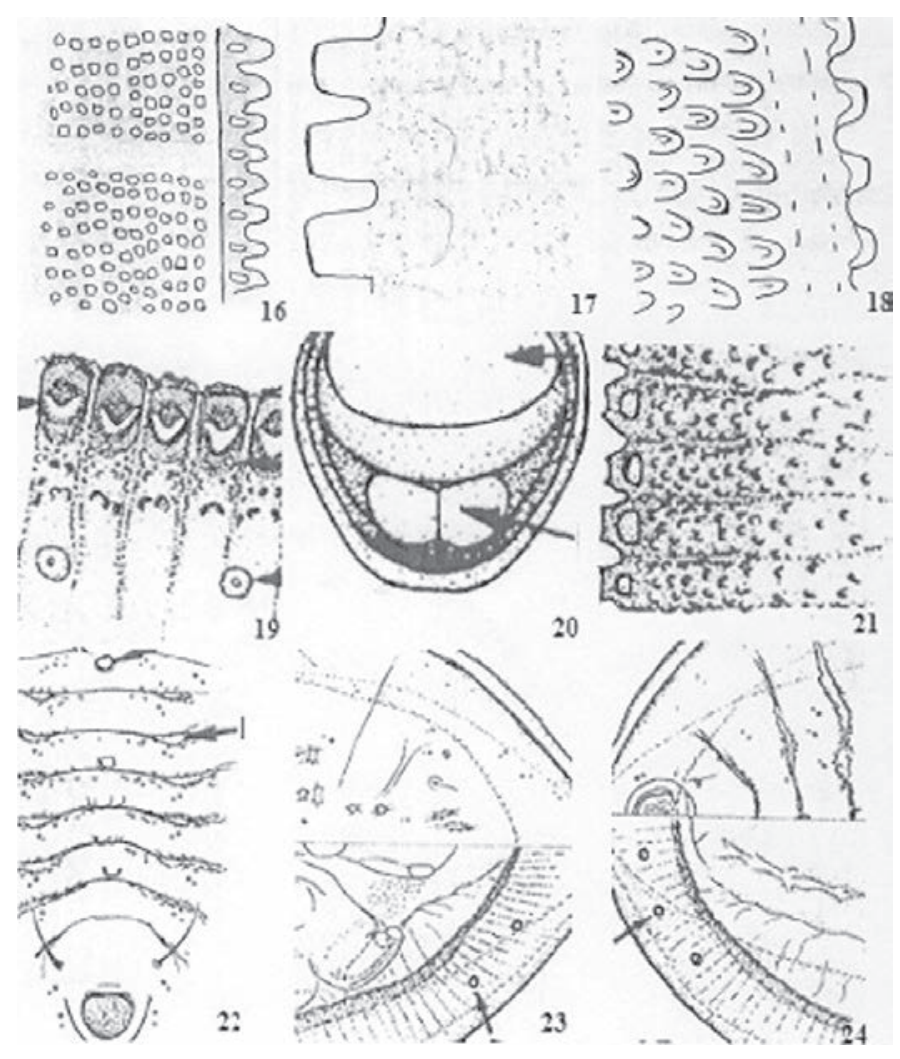

Figuras 16-24. (Sampson y Drews, 1941); 19-24 (Nakahara, 1995). 16) Area marginal de T. quadratus, 17) Área marginal de T. truncatus, 18) Área marginal de T. contigua. 19) Margen de T. caulicola, 20) Orificio vasiforme de T. bidentatus., 21) Margen de T. bidentatus. 22) Abdomen de T. acaciae, 23, 24) Área cefálica y caudal de $T$. birreflexa.

tava seta abdominal más cerca del orificio que de la sutura VIII, la cual se prolonga hacia la parte lateral (Fig. 19) .................. T. caulicola Nakahara 7 Orificio vasiforme sin una proyección en forma de "U" ligeramente elevado en el segmento abdominal VIII,orificio anterior al margen por 3/4 sulongitud, sin depresiones abdominales (Fig. 19) ................... T. mexicana Nakahara - Orificio vasiforme con una proyección en forma de "U” ligeramente elevado en el segmento abdominal VIII, orificio anterior al margen por poco más que su propia longitud, con depresiones abdominales medias (Fig. 22) ................... acaciae (Quaintance) 8 Cefalotórax sin setas medianas, octava seta abdominal ausente o en la parte posterolateral del orificio vasifor-

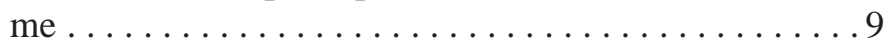
- Cefalotórax con 1-3 setas medianas, octava seta abdominal en la parte anterolateral del orificio vasiforme ....11 
9 Orificio vasiforme con celdas; tubérculos pequeños con glándulas pálidas en la parte posterolateral del orificio vasiforme; octava seta abdominal ausente . . . . . . . . 10 - Orificio vasiforme sin celdas; octava seta abdominal presente (Fig. 2) .......T. quadratus Sampson y Drews 10 Orificio vasiforme con una hilera de 3-4 celdas en cada lado; seta caudal sobre el $1 / 4$ distal del submargen sobre varios hospederos. (Fig. 33) .... . T. usorum (Cockerell) - Orificio vasiforme con dos hileras en cada lado y una posterior totalizando cerca de 15-16 celdas; seta caudal sobre la mitad submargen sobre Quercus. (Fig. 32)................. T. quercicola Nakahara 11 Seta cefálica presente; submargen con áreas glandulares pálidas triangulares alargadas en la base de los dientes marginales....................... 12 - Seta cefálica ausente; submargen con áreas glandulares pálidas en forma de $\mathrm{V}$ o U en la base de los dientes marginales........................... 15

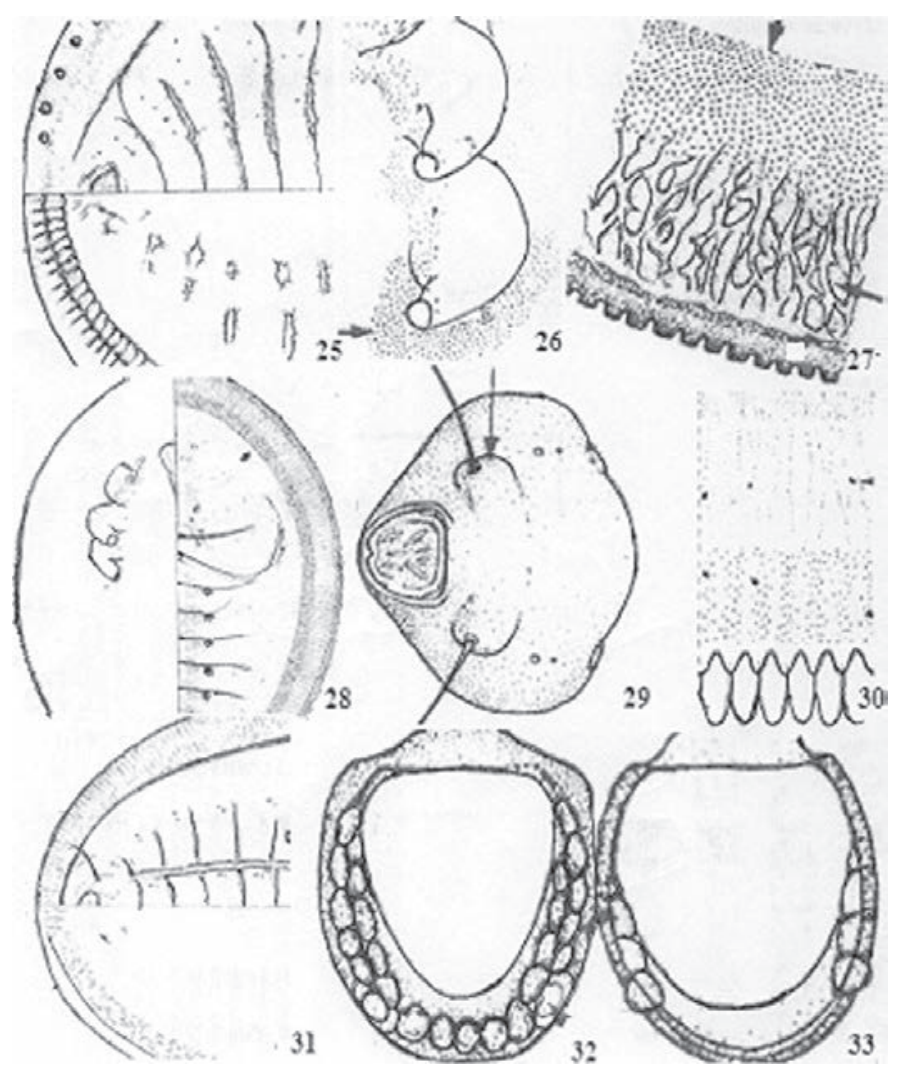

Figuras 25-33 (Nakahara, 1995). 25) Abdomen de T. mexicana. 26) Patas de T. chivela. 27) Submargen ventral de T. chivela. 28) Pupario de T. perseae. 29) Orificio vasiforme de T. perseae. 30) Margen y submargen de $T$. perseae. 31) Abdomen de T. pringle. 32) Orificio vasiforme de T. quercicola. 33) Orificio vasiforme de T. usorum.
12 Espínulas ausentes de la base de las patas torácicas; poros del disco grandes de 5-7 $\mu \mathrm{m}$ de diámetro alineado en una hilera regular sobre el 1/3 proximal del submargen; segmentos abdominales con tubérculos medios. Sobre Quercus. . . . . . . . . . . . . . . . . . . . 13 - Espínulas presentes de la base de las patas torácicas; poros del disco pequeños de 2-3 $\mu \mathrm{m}$ de diámetro alineado en una hilera con espaciado irregular sobre el 1/3 proximal del submargen; segmentos abdominales con tubérculos medios; sobre varios hospederos . ...........14 13 Marcas oculares difusas; áreas glandulares ovales pálidas en el submargen, tan largas como los dientes marginales; dos tubérculos anteriores del orificio vasiforme ................... perileuca (Cockerell) - Marcas oculares ausentes; áreas glandulares alargadas pálidas en el submargen, 1.5 veces tan largas como los dientes marginales; tubérculos anteriores del orificio vasiforme ausentes; orificio vasiforme muy elevado. ................ T. melanops (Cockerell) 14 Área subventral del abdomen con espínulas; vientre submarginal con áreas ovales o irregulares; base del orificio vasiforme con una área abierta (Fig. 16, 17) ................... T. chivela Nakahara - Área subventral del abdomen sin espínulas; vientre submarginal sin áreas ovales o irregulares; base del orificio vasiforme con dos áreas abiertas separadas con un esclerito en la parte posterior y una en la parte anterior (Fig. 20, 21)............ T. bidentatus Sampson y Drews 15 Opérculo con una muesca pequeña en la parte posterior media, abdomen con depresiones medianas (Figs. 28-30) ................. T. perseae Nakahara - Opérculo sin una muesca pequeña en la parte posterior media, abdomen sin depresiones medianas . . . . . . 16 16 Área media del abdomen delineada por un surco en cada lado; grupo de poros subdorsales segmento abdominal IV ausentes; banda ancha de espínulas en ambos lados de las patas que se extienden en el abdomen......... 17 - Área media del abdomen no delineada por un surco en cada lado; con un grupo de 3-8 poros subdorsales de segmento abdominal IV; banda estrecha de espínulas en la base de las patas ........... T. mori (Quaintance) 17 Sutura transversa de la muda con pequeñas muescas; espínulas ventrales presentes en el abdomen; áreas oculares ovales transversas .......T. fici Quaintance y Baker - Sutura transversa de la muda sin pequeñas muescas; espínulas ventrales presentes solo en la parte anterior del abdomen; áreas oculares ovales o semicirculares (Fig. 31)............. T. pringlei Quaintance y Baker 
AGRADECIMIENTOS. A Martin J. H., por los artículos proporcionados y por comentarios sobre esta especie.

\section{LITERATURA CITADA}

Baker, J. M. (1937). Notes on some Mexican Aleyrodidae. Anales del Instituto de Biología de la Universidad Nacional Autónoma de México, 8, 599-629.

Carapia-Ruiz, V. E., Sánchez-Flores, O. A., Pochotitla-Campos, I., \& Castillo-Gutiérrez, A. (2016). Estudio del género Tetraleurodes cockerell (Hemiptera: Aleyrodidae) de México. Entomología Mexicana, 3, 836-842.
Martin, J. H. (2004). Whiteflies of Belize (Homotera: Aleyrodidae) Part 1-Introdution and account of the subfamily Aleurodicinae Quaintance \& Baker. Zootaxa, 681, 1-119.

Martin, J. H. (2005). Whiteflies of Belize (Homotera: Aleyrodidae) Part 2- a review of the subfamily-Aleurodinae Quaintance \& Baker. Zootaxa, 681, 1-119.

Nakahara S. (1995). Taxonomic studies of the genus Tetraleurodes (Homoptera: Aleyrodidae). Insecta mundi, 9, 105-150.

Quaintance, A. L. \& Baker, A. C. (1914). Classification of the Aleyrodidae. Part II. U.S.D.A. Bur. Ent. Tech. Ser., 27, 95-109.

Sampson, W. W. \& Drews, E. A. (1941). Fauna Mexicana IV. A review of the Aleyrodidae of México. Anales de la Escuela Nacional de Ciencias Biológicas, 2, 143-189. 